\title{
The effects of a plastic-film covering on vibrotactile pattern perception with the Optacon
}

\author{
ROGER W. CHOLEWIAK and AMY A. COLLINS \\ Princeton University, Princeton, New Jersey
}

\begin{abstract}
Three experiments were conducted to determine whether the perception of vibrotactile patterns presented to the fingertip on the Optacon (a reading machine for blind persons) would be affected by covering the array with a plastic membrane. Occasionally in psychophysical and physiological studies that involve the Optacon, the array is covered with a plastic film to minimize electrical transients during electrophysiological recording and to maintain cleanliness. In the three studies described here, observers performed a spatial acuity task, identified patterns of a previously learned set, and judged the loudness of vibrotactile noise patterns by means of absolute magnitude estimation, on both the covered and the uncovered array. Performance in the spatial acuity and pattern identification tasks was not affected by the presence of the protective film. The rate of growth of perceived intensity was also the same with and without the plastic film, although there was a slight increase in vibrotactile detection threshold with the film.
\end{abstract}

The Optacon is a commercially available reading machine for blind persons that consists of a solid-state camera connected to an array of vibrators designed to stimulate the distal pad of the left index finger. ${ }^{1}$ In normal use, the camera is swept over printed text, and corresponding patterns of vibration are generated on the $6 \times 24$ array of contactors (see Bliss, Katcher, Rogers, \& Shepard, 1970). In addition to its usefulness to visually disabled persons, the system, under computer control, has found use in numerous research laboratories, because its dense vibrotactile array can be applied to basic studies of two-dimensional cutaneous pattern processing (e.g., Cholewiak \& Craig, 1984; Craig, 1976, 1980; Evans \& Craig, 1986; Gardner, 1987; Schneider, Hughes, Epstein, \& Bach-y-Rita, 1986; Sherrick \& Cholewiak, 1987).

The Optacon has been used in both psychophysical and physiological studies (e.g., Gardner \& Palmer, 1989), allowing the model of vibrotactile pattern perception to encompass both behavior and neurophysiology. The strength of such composites of the results of parallel psychophysical and physiological studies depends on the equivalence of both procedures and methodology. The present experiments were prompted by a procedural variation noted in Gardner's physiological studies of receptor populations in the fingers of monkeys using the Optacon as the stimulator (e.g., Gardner, 1987). In such preparations, the Optacon display is typically covered with a sheet of flexible plastic to eliminate interference with electrophysiological recordings. An additional benefit is that the cover-

This research was supported by Grant NS-04775-26 from the National Institutes of Health to Princeton University and SBIR Contract N44-NS-8-2394 6/30/88 to Sensor Electronics, Mt. Laurel, NJ. Reprint requests should be addressed to either author at the Department of Psychology, Green Hall, Princeton University, Princeton, NJ 08544-1010. ing helps to keep the array clean. The contactors are on $2.54-\mathrm{mm}$ centers across its six columns, and on $1.27-\mathrm{mm}$ centers along its 24 rows. Each of the $0.25-\mathrm{mm}$ diameter contactors touches the skin through 1-mm holes in the protective fingerplate. Because of the close tolerances in the system, debris, including skin cells and lint, can readily clog the openings and limit proper operation. Professional cleaning, which is both costly and time consuming, is then required to restore reliable operation.

In order to be confident that the presence of the plastic film does not violate the parallelism between the two classes of experiments, its influence should be examined in detail. Reports from Gardner's laboratory (Gardner \& Palmer, 1989) suggest that the plastic membrane does not affect judgments of vibrotactile movement perception made by human subjects. However, because the covering could influence the results in a variety of ways, its effects should be evaluated in several different paradigms. The three studies to be described here were designed to examine the consequences of covering the Optacon display with a commonly available plastic film, Saran Wrap (Dow Chemical Corp.). We compared vibrotactile acuity, vibrotactile pattern identification performance, and magnitude estimation of the loudness of vibrotactile noise stimuli, with and without the protective film.

Saran Wrap is a polyvinylidene chloride plastic sheet with a measured thickness of $1.0 \mathrm{mil}(0.0254 \mathrm{~mm})$. It was chosen as the membrane material because it is a relatively heavy kitchen wrap, and because it is readily available to other laboratories for replication or extension of the present study. In all of the experiments to be described, a piece of the membrane that measured approximately $4 \times 5$ in. was placed in the Optacon finger rest as required, completely covering the vibrotactile array. The observer rested the fingers of the left hand on top of the sheet, positioning the index finger on the display itself. The observer 
was also instructed to use care in placing the finger, so as to avoid wrinkling the film in the region over the array.

\section{EXPERIMENT 1}

In the first comparison, we employed a measure of tactile acuity that is comparable to visual minimum separable acuity. The patterns consisted of active elements in one or two rows of the Optacon array. In the case of the patterns consisting of elements in two rows (" 2 " patterns), the rows were either adjacent or separated by one through four inactive rows. Every other element was turned on in the active rows, so the resulting pattern was W-shaped and made up of six vibrators. These were compared against the single row patterns (" 1 " patterns), which had six active elements in a straight line. Since both patterns had the same number of elements, loudness cues should not have influenced the judgments.

Observers were required to identify whether a pattern was made up of one or two rows by pressing keys marked " 1 " or " 2 ." Half of the stimuli were in each category, and the location of the pattern on the array was varied randomly from trial to trial. Patterns were turned on for a duration of either 26 or $200 \mathrm{msec}$. In these and the following experiments, the loci of active stimulus elements were restricted to the upper 18 rows of the Optacon in order to avoid the distal crease of the finger that might not contact the array completely.

Six trained observers judged the patterns on the uncovered array in two series of 200 trials. Trials were blocked by duration, while the separation, ranging from zero (a single row) to four inactive rows, varied within each block. Two of these observers were retested with the plastic film covering the array. The data for the 26-msec duration patterns are presented in Figure 1 as percent correct identifications as a function of separation of the active rows.

For clarity, only the 26-msec duration data are shown in Figure 1, but those for the 200-msec duration patterns were essentially identical. No significant difference was found between the covered and uncovered conditions. Threshold for these patterns, as measured by the separation at which $75 \%$ of the correct responses occurred, ranged from 3.6 to 3.8 rows $(4.6-4.8 \mathrm{~mm})$, which, of course, cannot be resolved on the Optacon. The 200-msec patterns had a slightly smaller threshold than did the 26-msec patterns, which was to be expected, because patterns are better elaborated with increasing duration (Craig, 1980).

\section{EXPERIMENT 2}

In the next experiment, we compared the accuracy for identifying a set of 16 vibrotactile patterns presented with either a covered or an uncovered array. This particular pattern set has been used to examine the kinds of interaction possible among vibrotactile patterns in recognition and masking paradigms in this laboratory and in others (Craig \& Evans, 1987; Evans, 1987; Evans \& Craig, 1986; Sherrick \& Cholewiak, 1987, 1988a, 1988b). The set, visually represented in Figure 2, consists of all combinations of four line segments (including the null or blank combination) presented on the Optacon. Each vertical line represents activity along one column of 18 vibrating pins, while each horizontal line represents activity across two adjacent rows of 6 pins.

Seven paid observers were trained to identify the members of the 16-pattern set. Sessions consisted of a quasirandom series of 240 trials (each pattern presented 15

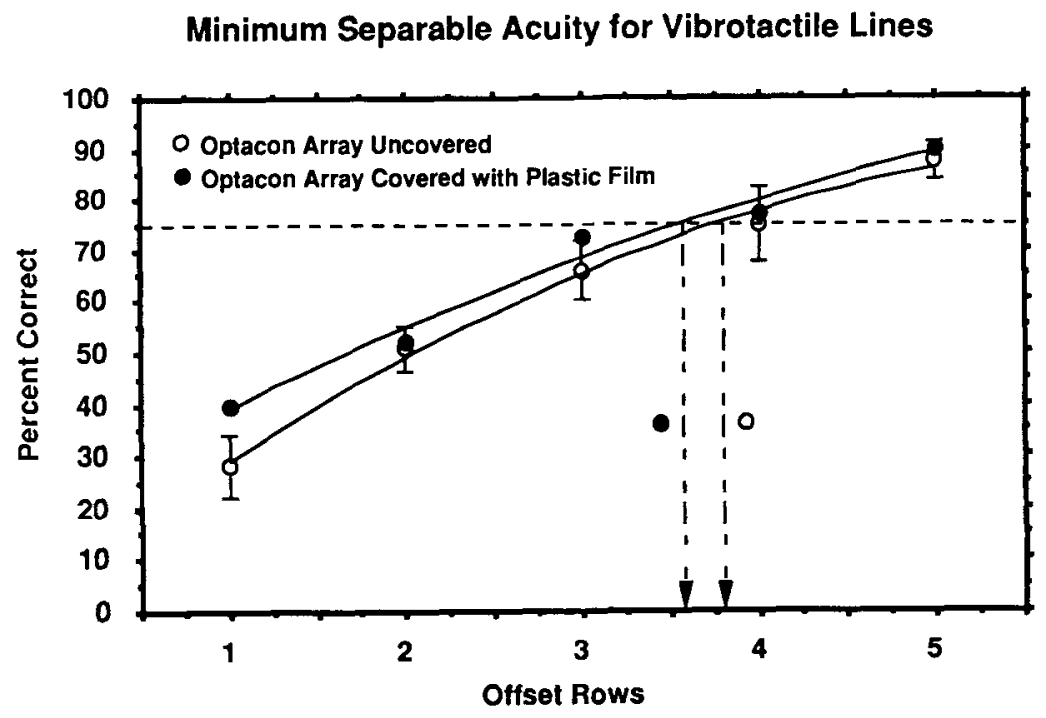

Figure 1. Minimum separable acuity for patterns consisting of pairs of three-element rows on the Optacon array, as a function of the position of the second row in the pair (offset). Threshold acuity, as defined by the separation at which $75 \%$ of the correct responses occur, is indicated by the dropped arrows for conditions in which the array was either uncovered or covered with plastic film. 


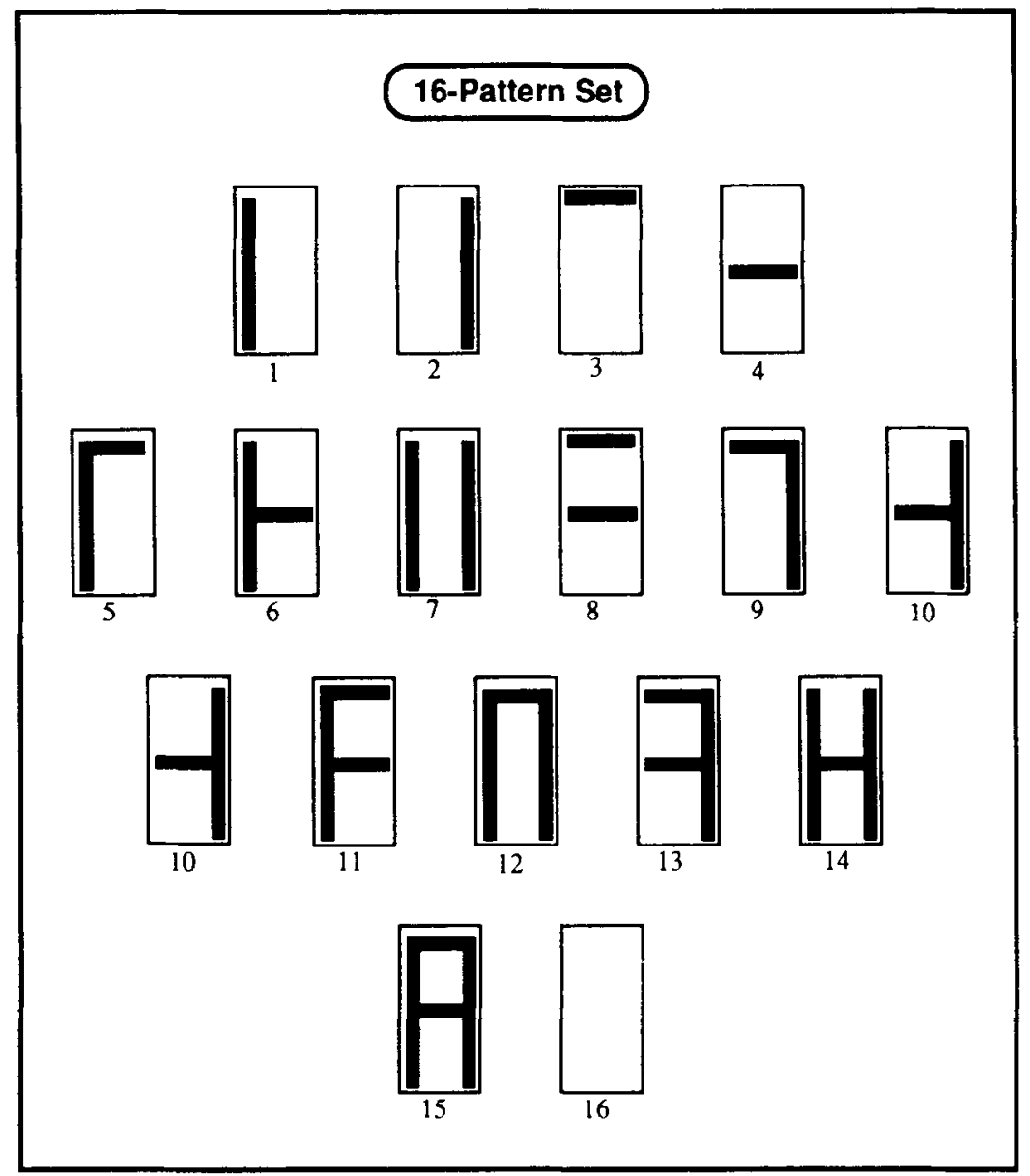

Figure 2. Visual representations of the 16-pattern set. Each pattern contains zero, one, two, three, or four of the line segments. A line consists of either a single column (18 vibrators) or a pair of rows (12 vibrators).

times) divided into blocks of 48 trials. The patterns were presented for a duration of $26 \mathrm{msec}$. On each trial, observers were required to feel the pattern and then strike a numbered key corresponding to the identification number shown in Figure 2. Visual representations, like those in the figure, were always available, and trial-bytrial feedback was provided. The results are shown in Figure $3 \mathrm{~A}$. The high initial performance rates are, to a large degree, owing to the presence of the visual representations. In other studies, when the pictures were not available, we have seen as much as a $30 \%-40 \%$ drop in initial rates (Cholewiak, Collins, \& Sherrick, 1989). Most observers showed asymptotic performance in the sixth training session, at which time mean performance was $84 \%$ correct identifications (range $=70 \%-97 \%$ ). After asymptote was reached, each observer completed another session in which the same procedures were followed, except that the plastic film covered the Optacon display. When performance in this session was compared with that in the sixth acquisition session, no significant differences in identification performance were found (Wilcoxon signed-rank test, $z=0, p>.05$ ).
We also performed an analysis to measure the number of line segments in the response as a function of the number of line segments in the stimulus. This analysis reveals whether the perceived pattern contained as much spatial information as was presented in the stimulus. In all cases, the presence of the plastic film did not affect performance ( $z$ ranged from -0.34 to $-1.35, p>.05$ ) These comparisons are shown in Figure 3B. Parenthetically, one observer tried an additional identification session wearing a latex surgical glove (measured thickness over the finger $=4.0$ mils, or $0.10 \mathrm{~mm}$ ). Again, no difference was found in identification performance with and without the glove.

\section{EXPERIMENT 3}

Finally, we examined judgments of pattern loudness as a function of pattern intensity with and without the interposed plastic film. We varied the intensity on the Optacon by remotely changing the driving voltage to the piezoceramic pins (see Sherrick \& Cholewiak, 1988b, for a description of the technique). The stimuli were patterns 

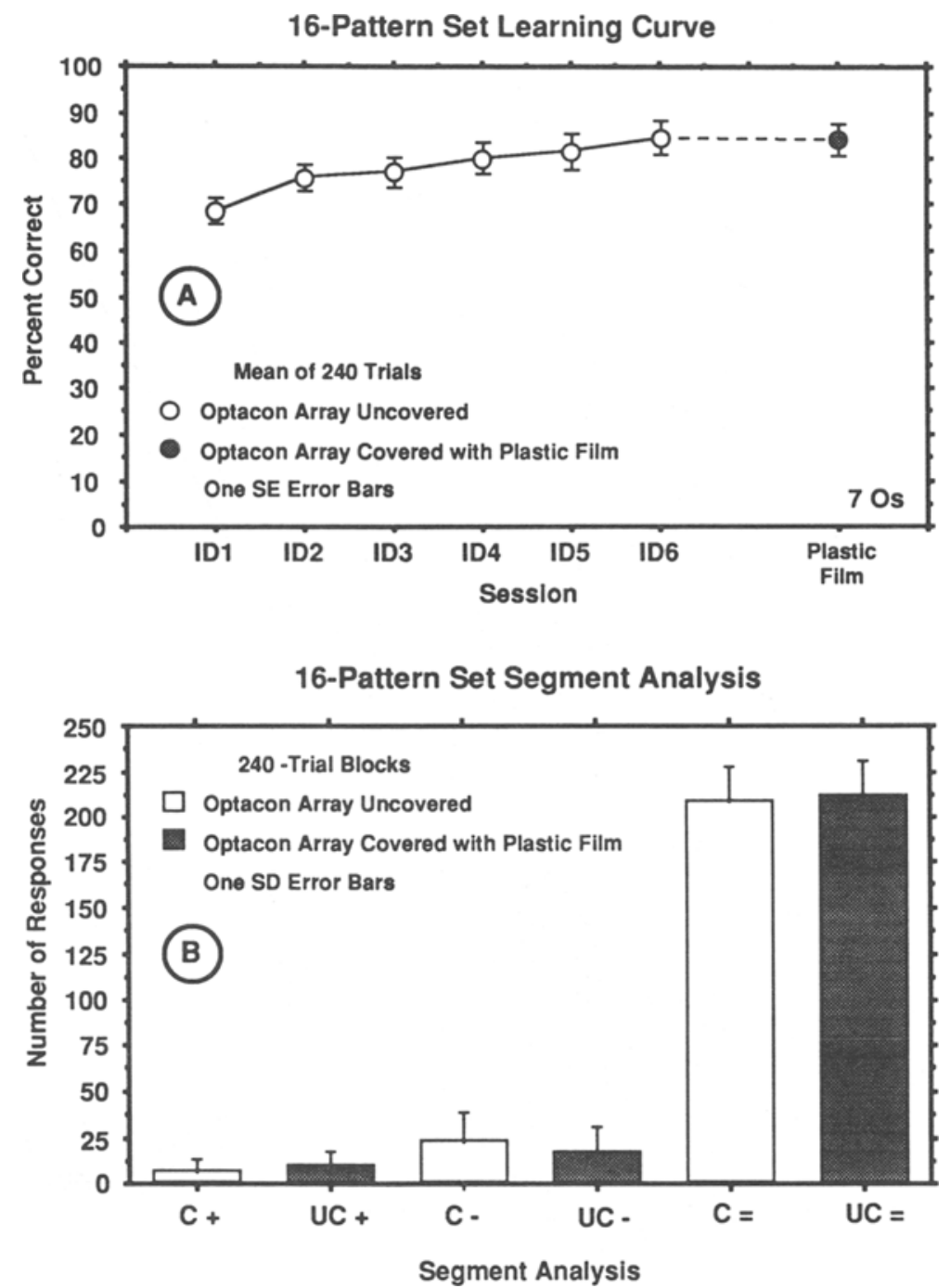

Figure 3. Identification performance for the 16-pattern set with and without plastic film covering the Optacon array. The upper panel (A) shows the learning curve for the patterns, with a direct comparison between the sixth training session (ID6) and a session with plastic film. The lower panel (B) shows the results of the segment analysis, in which responses were categorized into those with more $(+)$, fewer $(-)$, or the same $(=)$ number of segments as the stimulus. No significant differences were found in any of these comparisons between the covered (C) and uncovered (UC) conditions.

made up of two densities of vibrotactile noise generated on the Optacon. One (the high-density noise pattern) was made up of all 108 elements in the top $6 \times 18$ array of elements repeated six times over the 26 -msec duration of the stimulus. The second (low-density noise) was generated by displaying six different sets of 31 randomly chosen elements ( $28 \%$ of the total) over the $26-\mathrm{msec}$ pattern duration.

Judgments were made according to the method of absolute magnitude estimation. Six observers served in two sessions in which the growth of loudness of the noise patterns was examined. In one session, the plastic covering the array was present; in the other, it was not. In each session, the observers were required to (1) participate in a brief training exercise involving judgments of visual stimuli; (2) serve in a threshold determination series; and (3) judge three blocks of 20 stimuli presented in a quasirandom order. The training series was conducted to familiarize observers with the method of magnitude estimation in a situation with some degree of familiaritynamely, judging line lengths presented visually (see, e.g., Stevens, 1975, p. 30). Thresholds for detecting vibratory stimuli were taken with the method of adjustment on the low-density noise patterns repeating once every second.

Observers were then presented with stimuli that ranged in 2- $\mathrm{dB}$ increments in voltage from threshold for the lowdensity noise stimuli to a maximum of about $20 \mathrm{~dB}$ re that level. Because of equipment limitations, some of the 


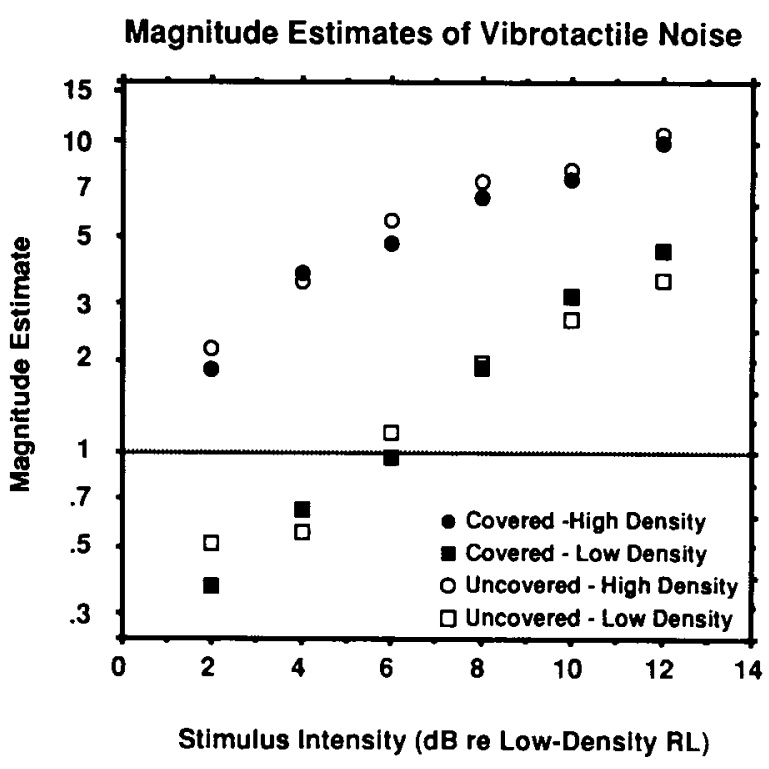

Figure 4. Magnitude estimates of high- and low-density noise patterns on the Optacon array. Data are shown for conditions in which the display was either covered with plastic film or uncovered. The high-density patterns consisted of all 108 vibrators in the $6 \times 18$ array, while the low-density noise patterns were made up of 31 of these. Thresholds were determined with the low-density patterns, and the intensities of the stimuli are defined with reference to those thresholds. There are no significant differences between the data from the uncovered and covered conditions.

higher intensity stimuli could not be presented if an observer's threshold was too high. Not surprisingly, measured thresholds were significantly higher when the plastic film was interposed between the finger and the array $[-7.4 \mathrm{~V}$ vs. $-8.3 \mathrm{~V}$ on the Optacon, which has a maximum of $-45 \mathrm{~V}, t(5)=2.77, p<.05$ ]. Although statistically significant, the difference represents less than $2 \mathrm{~dB}$ of loudness. Three repetitions of each intensity level were presented, with those in the first block considered to be practice (see, e.g., Hellman \& Meiselman, 1988). Geometric means of the estimates from the second and third blocks were averaged across observers for each condition. No significant differences were found between the estimates of pattern loudness with and without the covering in both the high- and the low-density noise conditions [high-density noise: $t(7)=-0.6, p>.05$; low-density noise: $t(7)=0.77, p>.05$ ]. These data are shown in Figure 4.

\section{DISCUSSION}

Covering the Optacon array with plastic film did not interfere with vibrotactile pattern acuity, identification, or loudness in these studies. These results were somewhat unexpected. We assumed that some degree of "blurring" of patterns might occur. To the contrary, observers often suggested that there appeared to be some "sharpening" of the sensation when the plastic film was present. An intensification of sensation in similar circumstances has been recorded by Green (1981) and others. Green examined a phenomenon he termed the "paper effect," in which, under some conditions, the interposition of a layer of paper could modify the intensitive judgments of the roughness of a surface felt by the index finger. In fact, by our measures, neither sharpening nor blurring actually occurred. Overall, no differences were seen with these tasks between the two conditions.

The only difference that we did find between the two conditions occurred in the amplitude of vibration at the finger required for vibrotactile detection threshold. Threshold was slightly higher when the plastic film was present. This is not surprising, because the amplitude of the stimulus produced by the piezoceramic benders would likely be attenuated by the presence of the plastic membrane. Our threshold measures bear out this assumption.

There are still other possible ways in which the film might affect performance. For example, acquisition of a pattern set might be influenced by the film, even though the asymptotic levels are not. We suspect, however, that any influence would be minimal, because patterns were not made less distinct by the presence of the film, as is evidenced by the lack of an effect on our acuity measure.

At a practical level, we have used the film in several extended studies of vibrotactile pattern perception. The lifespan of the material seems to be considerable. In one study (Cholewiak et al., 1989), a single piece of Saran Wrap was used over a period of 2 months, for more than 100 sessions. Observers did not report any changes in sensation either within or over sessions. The major complaint was that it was more difficult to move the finger on the array with the plastic, because it tended to adhere to the fingertip.

Our findings suggest that the conclusions from parallel studies in psychophysical and electrophysiological laboratories can be considered compatible even if the procedures differ regarding the presence or absence of a plastic-film covering on the Optacon. We find no evidence to suggest that such a covering could not be used routinely. Covering the array prevents it from being fouled with the dirt and dust that results from many observers' using the device over an extended period of time. This could eliminate the need for an annual cleaning that results in system down-time, high costs, and/or array replacement because of this type of damage.

\section{REFERENCES}

Bliss, J. C., Katcher, M. H., Rogers, C. H., \& Shepard, R. P. (1970). Optical-to-tactile image conversion for the blind. In J. C. Bliss (Ed.), Tactile Displays Conference [Special issue]. IEEE Transactions on Man-Machine Systems, MMS-11, 58-64.

Cholewiak, R. W., Collins, A. A., Sherrick, C. E. (1989, November). Shape as the sole cue for vibrotactile pattern identification. Paper presented at the annual meeting of The Psychonomic Society, Atlanta, GA.

Cholewiak, R. W., CRaig, J. C. (1984). Vibrotactile pattern recognition and discrimination at several body sites. Perception \& Psychophysics, 35, 503-514.

CraIG, J. C. (1976). Vibrotactile letter recognition: The effects of a masking stimulus. Perception \& Psychophysics, 20, 317-326. 
Craig, J. C. (1980). Modes of vibrotactile pattern generation. Joumal of Experimental Psychology: Human Perception \& Performance, 6 , 151-166.

CrajG, J. C., E Evans, P. M. (1987). Vibrotactile masking and the persistence of tactual features. Perception \& Psychophysics, 42, 309-317.

Evans, P. M. (1987). Vibrotactile masking: Temporal integration, persistence, and strengths of representations. Perception \& Psychophysics, 42, 515-525.

Evans, P. M., CRaig, J. C. (1986). Temporal integration and vibrotactile backward masking. Joumal of Experimental Psychology: Human Perception \& Performance, 12, 160-168.

GARDNER, E. P. (1987). Somatosensory cortical mechanisms of feature detection in tactile and kinesthetic discrimination. Canadian Journal of Physiology \& Pharmacology, 66, 439-454.

Gardner, E. P., \& Palmer, C. I. (1989). Simulation of motion on the skin: 1. Receptive fields and temporal frequency coding by cutaneous mechanoreceptors of OPTACON pulses delivered to the hand. Joumal of Neurophysiology, 62, 1410-1436.

GreEN, B. G. (1981). Tactile roughness and the "paper effect." Bulletin of the Psychonomic Society, 18, 155-158.

Hellman, R. P., Meiselman, C. H. (1988). Prediction of individual loudness exponents from cross-modality matching. Joumal of Speech \& Hearing Research, 31, 605-615.
Schneider, S. L., Hughes, B., Epstein, W., \& Bach-Y-Rita, P. (1986). The detection of length and orientation changes in dynamic vibrotactile patterns. Perception \& Psychophysics, 40, 290-300.

Sherrick, C. E., Cholewiak, R. W. (1987). Princeton Cutaneous Research Project (Report No. 50, December 1987). Princeton, NJ: Princeton University, Department of Psychology.

Sherrick, C. E., \& Cholewiak, R. W. (1988a). Princeton Cutaneous Research Project (Report No. 51, June 1988). Princeton, NJ: Princeton University, Department of Psychology.

Sherrick, C. E., \& Cholewiak, R. W. (1988b). Princeton Cutaneous Research Project (Report No. 52, December 1988). Princeton, NJ: Princeton University, Department of Psychology.

Stevens, S. S. (1975). Psychophysics. New York: Wiley.

\section{NOTE}

1. The Optacon is manufactured by Telesensory Systems, Incorporated, 455 North Bernardo Avenue, Mountain View, CA 94039-7455.

(Manuscript received October 23, 1989; revision accepted for publication December $27,1989$. 